\title{
Combined gene therapy with vascular endothelial growth factor plus apelin in a chronic cerebral hypoperfusion model in rats
}

\author{
Masafumi Hiramatsu, MD, ${ }^{1}$ Tomohito Hishikawa, MD, ${ }^{1}$ Koji Tokunaga, MD, ${ }^{2}$ Hiroyasu Kidoya, PhD, ${ }^{3}$ \\ Shingo Nishihiro, MD, ${ }^{1}$ Jun Haruma, MD, ${ }^{1}$ Tomohisa Shimizu, MD, ${ }^{1}$ Yuji Takasugi, MD,1 \\ Yukei Shinji, MD, ${ }^{1}$ Kenji Sugiu, MD, ${ }^{1}$ Nobuyuki Takakura, MD, ${ }^{3}$ and Isao Date, MD' \\ 'Department of Neurological Surgery, Okayama University Graduate School of Medicine, Dentistry and Pharmaceutical \\ Sciences; 'Department of Neurosurgery, Okayama City Hospital, Okayama; and ${ }^{3}$ Department of Signal Transduction, Research \\ Institute for Microbial Diseases, Osaka University, Suita, Japan
}

\begin{abstract}
OBJECTIVE The aim of this study was to evaluate whether combined gene therapy with vascular endothelial growth factor (VEGF) plus apelin during indirect vasoreconstructive surgery enhances brain angiogenesis in a chronic cerebral hypoperfusion model in rats.
\end{abstract}

METHODS A chronic cerebral hypoperfusion model induced by the permanent ligation of bilateral common carotid arteries (CCAs; a procedure herein referred to as "CCA occlusion" [CCAO]) in rats was employed in this study. Seven days after the CCAO procedure, the authors performed encephalo-myo-synangiosis (EMS) and injected plasmid(s) into each rat's temporal muscle. Rats were divided into 4 groups based on which plasmid was received (i.e., LacZ group, VEGF group, apelin group, and VEGF+apelin group). Protein levels in the cortex and attached muscle were assessed with enzyme-linked immunosorbent assay (ELISA) on Day 7 after EMS, while immunofluorescent analysis of cortical vessels was performed on Day 14 after EMS.

RESULTS The total number of blood vessels in the cortex on Day 14 after EMS was significantly larger in the VEGF group and the VEGF+apelin group than in the LacZ group ( $p<0.05$, respectively). Larger vessels appeared in the VEGF+apelin group than in the other groups $(p<0.05$, respectively). Apelin protein on Day 7 after EMS was not detected in the cortex for any of the groups. In the attached muscle, apelin protein was detected only in the apelin group and the VEGF+apelin group. Immunofluorescent analysis revealed that apelin and its receptor, APJ, were expressed on endothelial cells (ECs) 7 days after the CCAO.

CONCLUSIONS Combined gene therapy (VEGF plus apelin) during EMS in a chronic cerebral hypoperfusion model can enhance angiogenesis in rats. This treatment has the potential to be a feasible option in a clinical setting for patients with moyamoya disease.

https://thejns.org/doi/abs/10.3171/2016.8.JNS16366

KEY WORDS apelin; gene therapy; moyamoya disease; revascularization; vascular endothelial growth factor

$\mathrm{M}$ OYAMOYA disease (MMD) is a chronic, progressive cerebrovascular disease characterized by stenosis or occlusion of the bilateral supraclinoid internal carotid arteries and the development of an abnormal vascular network, called "moyamoya vessels," at the base of the brain that blocks cerebral flow. ${ }^{21}$ Indirect bypass surgeries such as enchepalo-myo-synangiosis (EMS) are mostly performed in pediatric patients with MMD. This is because indirect bypass surgery often results in insufficient collateral circulation for most adult and some pediatric patients. . $^{20,25,27,32,39}$

With the aim of improving collateral circulation after indirect bypass surgery, we previously investigated the effect of EMS combined with vascular endothelial growth

ABBREVIATIONS Ang-1 = angiopoietin-1; CBF = cerebral blood flow; CCA = common carotid artery; CCAO = CCA occlusion; EC = endothelial cells; ELISA = enzymelinked immunosorbent assay; EMS = encephalo-myo-synangiosis; MMD = moyamoya disease; $P B S=$ phosphate-buffered saline; $P F A=$ paraformaldehyde; RECA-1 = mouse monoclonal anti-EC antibody; VEGF = vascular endothelial growth factor.

SUBMITTED February 17, 2016. ACCEPTED August 24, 2016.

INCLUDE WHEN CITING Published online December 23, 2016; DOI: 10.3171/2016.8.JNS16366. 
factor (VEGF) gene administration into the temporal muscle in a chronic ischemia model in rats. ${ }^{15,22}$ Indirect bypass surgery for the treatment of patients with MMD was simulated by adding the EMS surgery to bilateral common carotid arteries (CCAs) ligation. The data demonstrated that EMS plus injection of human VEGF plasmid, compared with EMS alone, significantly increased angiogenesis in the cerebral cortex..$^{15,22}$ However, VEGF overexpression can introduce a risk of immature vessel formation that results in plasma leakage and angioma formation. $4,11,24,35$

Apelin has been identified as the endogenous ligand for APJ, an orphan G protein-coupled receptor that is expressed in the cardiovascular and central nervous systems. ${ }^{29}$ The apelin/APJ system is involved in a wide range of physiological activities, such as heart contractility and blood pressure regulation, ${ }^{6}$ appetite and drinking behavior, ${ }^{23}$ neuroprotection, ${ }^{30}$ and angiogenesis. ${ }^{7,14,17,18,26}$ It has been reported that apelin plus VEGF effectively induced the formation of functional vessels that were larger than those induced with VEGF alone in a hindlimb ischemia model. ${ }^{17}$

In this study, we evaluated whether combined gene therapy with VEGF plus apelin during indirect vasoreconstructive surgery enhances brain angiogenesis in a chronic cerebral hypoperfusion model in rats.

\section{Methods}

\section{Animals and Surgical Procedures}

All animal procedures performed in this study were specifically approved by the Institutional Animal Care and Use Committee of Okayama University Graduate School of Medicine, Dentistry and Pharmaceutical Sciences.

Adult male Wistar rats (9-11 weeks old, weighing $250-350 \mathrm{~g}$ ) were used for the experiments. With the rats under general anesthesia via $2.0 \%$ halothane in a mixture of $40 \%$ oxygen and $60 \%$ nitrous oxide gas, the CCAs were carefully separated from the sympathetic and vagus nerves using a ventrocervical incision. Bilateral CCAs were ligated with 3-0 silk sutures (herein referred to as "CCA occlusion" [CCAO]). The rats' body temperature was maintained close to $37^{\circ} \mathrm{C}$ throughout the procedure by using a heating pad. Sham operations involved a skin incision and exteriorization of the bilateral CCAs without ligation. An interval of 7 days was allowed for postoperative recovery (Fig. 1A).

Seven days after the CCAO procedure, some of the rats underwent EMS surgery (Fig. 1B). This 7-day waiting period between CCAO and EMS surgery was incorporated into the experimental design because $\mathrm{CCAO}$ reduces cerebral blood flow (CBF) to $35 \%-50 \%$ of control levels and because CBF starts to recover at 1 week. ${ }^{8,37}$ We thought that delaying EMS surgery beyond the initial CBF recovery period could have a negative effect on angiogenesis. This 7-day waiting period was also used in previous reports from other institutions developing similar models. While under general anesthesia via intraperitoneal administration of pentobarbital sodium $(45 \mathrm{mg} /$ $\mathrm{kg}$ ), the rats were placed in a stereotactic apparatus with the top of the skull positioned horizontally. After a mid- line linear incision was made, the right temporal muscle was detached from the temporal bone. Craniotomy was then performed in the temporoparietal region using a dental drill. The dura mater was carefully opened and removed with no disruption of the brain surface (Fig. 1C). The exposed brain surface was covered with the muscle flap (Fig. 1D). Transfection of plasmid into the temporal muscle was performed using GenomOne-Neo transfection reagent (Ishihara Sangyo) according to the manufacturer's protocol. Rats were divided into 4 groups based on which plasmid(s) they received (i.e., LacZ group, VEGF group, apelin group, and VEGF+apelin group ). Each rat received $25 \mu \mathrm{g}$ of plasmid. In a previous study, we found a significant increase in capillary density simply by injecting $50 \mu \mathrm{g}$ of naked VEGF plasmid (without use of transfection reagent) into the temporal muscle. ${ }^{22}$ Moreover, we performed an optimal dose analysis that demonstrated a maximal angiogenic effect occurring with a $100-\mu \mathrm{g}$ dose of VEGF plasmid..$^{15}$ As a reference, we used a previous study from another group comparing transfection efficacy between the injection of naked plasmids versus the use of GenomOne-Neo reagent. That study found a 4 times higher transfection efficiency using the GenomOne-Neo method. ${ }^{38}$

\section{Immunofluorescence}

CCAO and sham rats were killed with an overdose of pentobarbital $(100 \mathrm{mg} / \mathrm{kg}) 1$ week after the CCAO or sham procedure (Fig. 1A); CCAO+EMS rats were killed 2 or 3 weeks after the CCAO procedure (1-2 weeks after EMS; Fig. 1B). The rats were perfused transcardially with 200 $\mathrm{ml}$ of cold phosphate-buffered saline (PBS) and $100 \mathrm{ml}$ of $4 \%$ paraformaldehyde (PFA) in PBS. The brain and transfected temporal muscle were removed and postfixed in the same fixative overnight at $4^{\circ} \mathrm{C}$ and subsequently stored in $30 \%$ sucrose in PBS until completely submerged. Frozen coronal sections (17 $\mu \mathrm{m}$ thick) were cut from each specimen on a cryostat, and the sections were thaw mounted on slides. Slides from the CCAO or sham operation without EMS surgery were evaluated by immunofluorescent analysis of endothelial cells (ECs) and apelin/APJ protein. Slides from the CCAO+EMS rat tissue were evaluated with immunofluorescent analysis of ECs. There were 8 slides from each group. Sections that included cortical surfaces were photographed at $10 \times$ magnification. The total number of vessels per field, percentage of vessel area per field, and number of large $(>10 \mu \mathrm{m})$ vessels per field were calculated for each image using ImageJ software (National Institutes of Health).

For the immunofluorescent staining of ECs, after several rinses in PBS, the slides were incubated in $10 \%$ fetal bovine serum in PBS for 1 hour. The slides were then washed and incubated with an affinity-purified mouse monoclonal anti-EC antibody (RECA-1, Abcam) with $1 \%$ fetal bovine serum for 2 hours at room temperature. The slides were washed and then incubated for 1 hour with a Cy3 anti-mouse IgG antibody (1:200 dilution) at room temperature.

For the immunofluorescent staining of APJ and ECs, after several rinses in PBS, slides were incubated in $10 \%$ fetal bovine serum in PBS for 1 hour. The slides were then washed and incubated with RECA-1 and an affinity-puri- 
$\begin{array}{lllllll}\text { A } & \text { Day-0 } & 7 & \text { B } & \text { Day-0 } & 7 & 14\end{array}$

sham

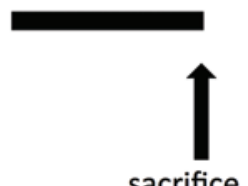

sacrifice

CCAO

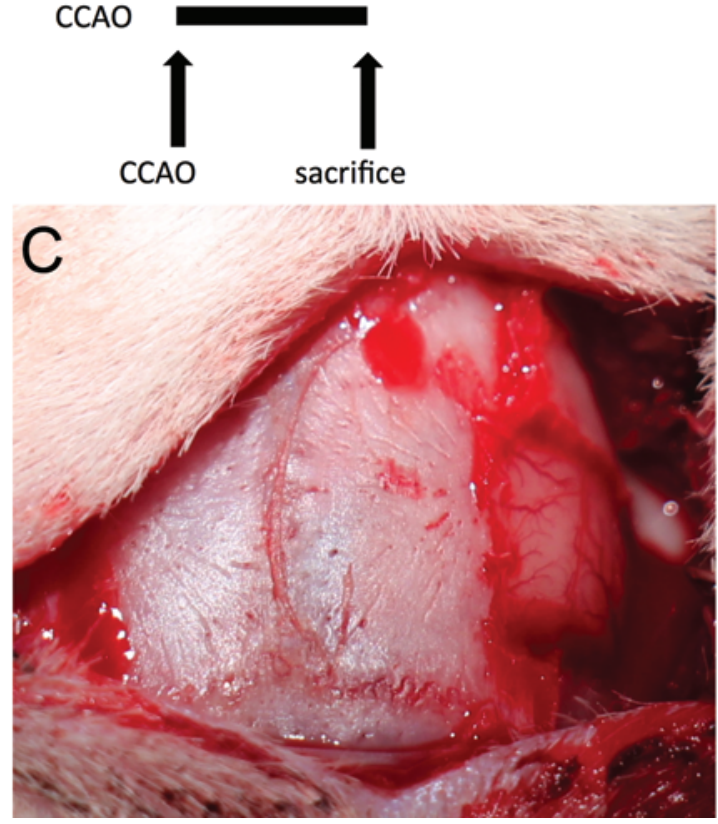

bypass model

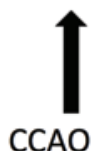

CCAO

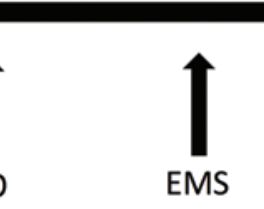

$+$

\section{plasmid injection}

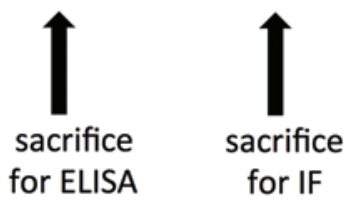

for IF

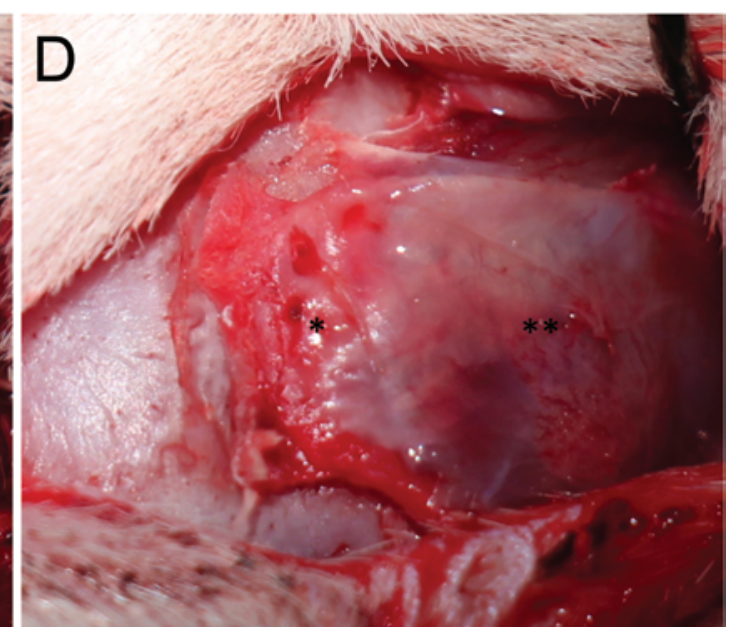

FIG. 1. Time course of the experimental design and indirect bypass surgery. A: Scheme showing the experimental design for the CCAO model. B: Scheme showing the experimental design for the indirect bypass surgery model. C: Exposed brain after craniotomy in the right temporoparietal region. D: Fascia $\left(^{*}\right)$ attached to the remaining parietal bone and brain covered with the temporal muscle $\left({ }^{*}\right)$. IF = immunofluorescence.

fied rabbit polyclonal anti-APJ antibody with $1 \%$ fetal bovine serum for 2 hours at room temperature. Anti-APJ antibody was provided by Dr. Kidoya, which was described previously. ${ }^{18}$ The slides were washed and then incubated for 1 hour with a Cy3 anti-mouse IgG antibody (1:200 dilution) and Alexa Fluor anti-rabbit IgG antibody (1:200 dilution) at room temperature.

For the immunofluorescent staining of apelin and ECs, after several rinses in PBS, slides were incubated in $10 \%$ normal goat serum in PBS for 1 hour. The slides were then washed and incubated with an affinity-purified rabbit polyclonal anti-von Willebrand factor antibody and an affinity-purified mouse monoclonal anti-apelin antibody with $1 \%$ normal goat serum for 2 hours at room temperature. Anti-apelin antibody was provided by Dr. Kidoya, which was described previously. ${ }^{18}$ The slides were washed and incubated for 1 hour with an FITC anti-rabbit IgG antibody (1:300 dilution) and a $\mathrm{Cy} 3$ anti-mouse $\mathrm{IgG}$ antibody (1:300 dilution) at room temperature.

\section{Enzyme-Linked Immunosorbent Assay Analyses}

One week after EMS surgery, CCAO+EMS rats $(n=$ 2 per group) anesthetized with an overdose of pentobarbital $(100 \mathrm{mg} / \mathrm{kg}$, intraperitoneal) were decapitated, and their brains and muscle tissues were quickly harvested. Brain and muscle were sliced at a $2 \mathrm{~mm}$ thickness. Brain cortex was obtained using a biopsy punch (3-mm hole, Kai Corp. and Kai Industries Co., Ltd.). The tissues were then homogenized in T-PER Tissue Protein Extraction Reagent (ThermoFisher Scientific Inc.) and processed in a centrifuge at $10,000 \mathrm{~g}$ for 10 minutes at $4^{\circ} \mathrm{C}$, retaining the supernatant. VEGF and apelin protein levels were measured using human VEGF enzyme-linked immunosorbent assay (ELISA; Immuno-Biological Laboratories Co., Ltd) and apelin-12 ELISA kits (Phoenix Pharmaceuticals, Inc.).

\section{Statistical Analyses}

The total number of blood vessels and the VEGF, apelin, and LacZ protein levels were evaluated statistically using single ANOVA, with a subsequent post hoc TukeyKramer test. Statistical significance was set at $p<0.05$.

\section{Results}

At the capillary level, the total number of blood vessels 2 weeks after EMS was significantly higher in the VEGF and VEGF+apelin groups than in the LacZ group 


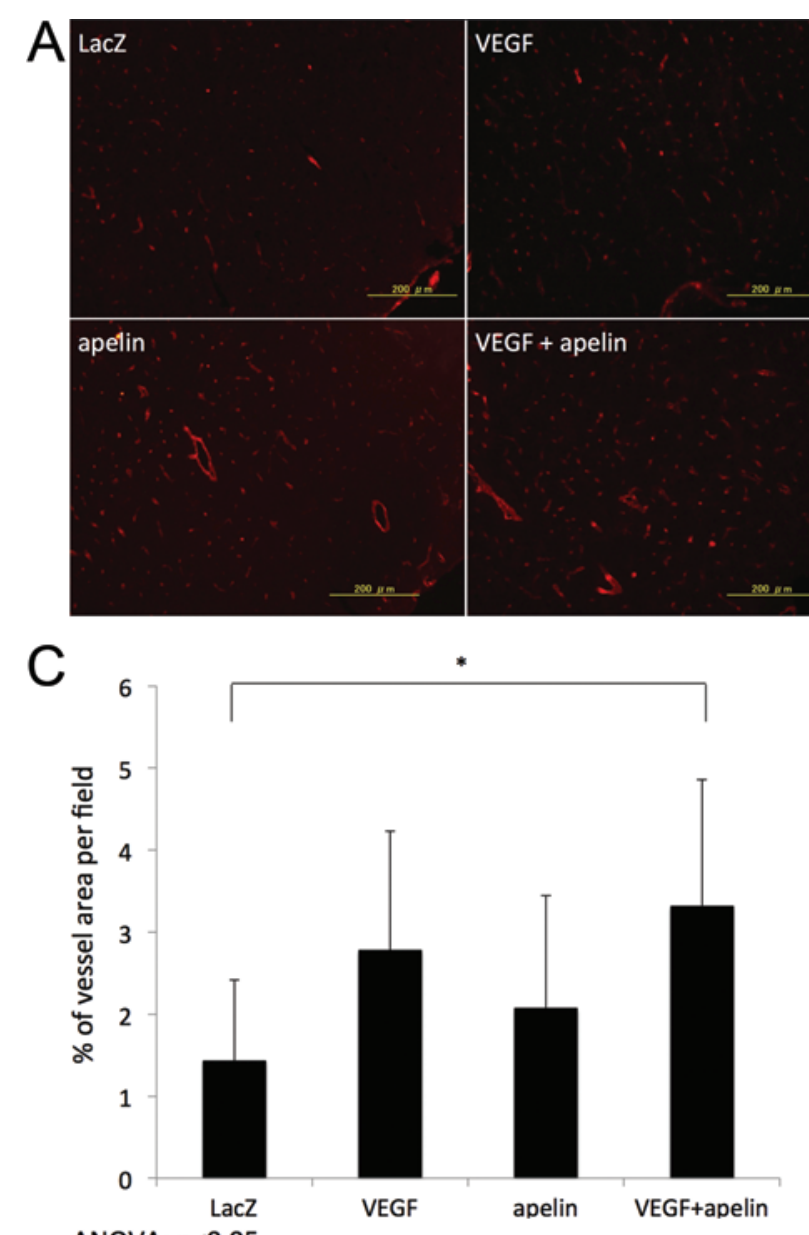

ANOVA: $p<0.05$

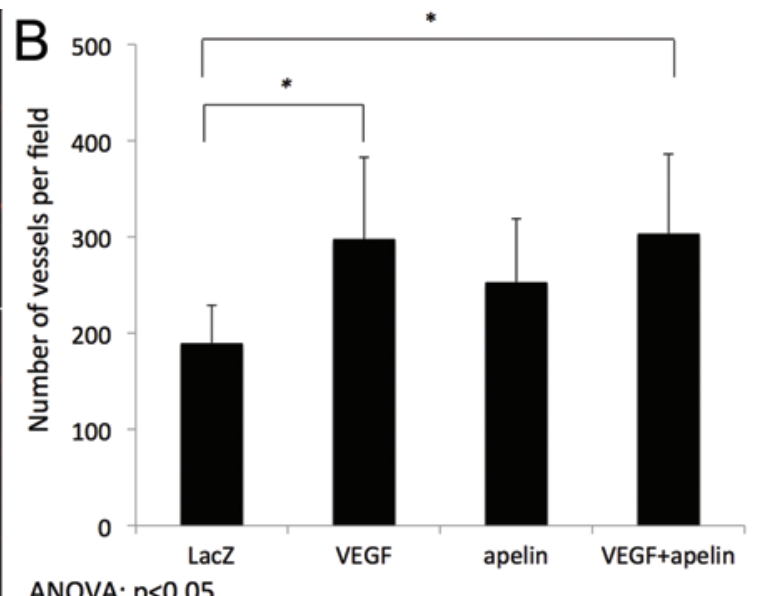

ANOVA: $p<0.05$

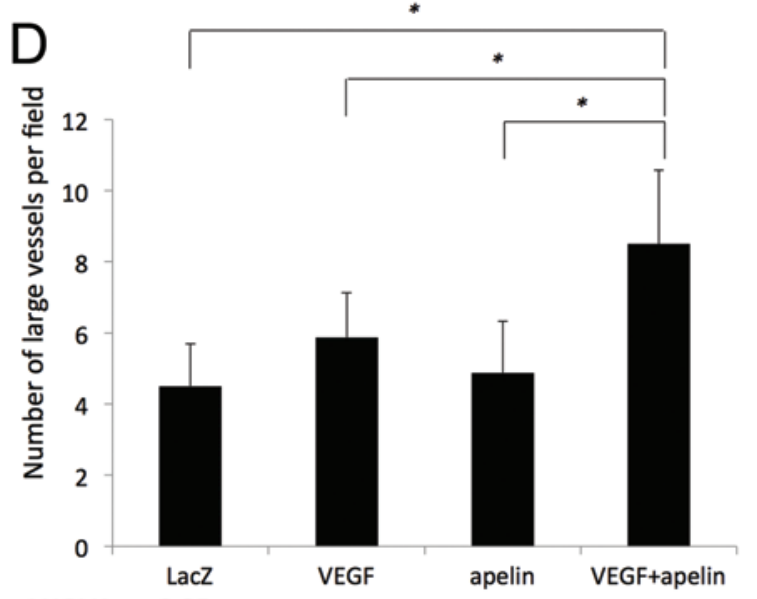

FIG. 2. Analysis of angiogenesis in an indirect bypass surgery model. A: RECA-1 staining of the cortex in the 4 groups. B: Quantitative evaluation of the number of vessels $\left({ }^{*} p<0.05\right)$. C: Percentage of vessel area per field $\left({ }^{*} p<0.05\right)$. D: Quantitative evaluation of the number of large $(>10 \mu \mathrm{m})$ vessels $\left({ }^{*} p<0.05\right)$.

( $p<0.05$, respectively; Fig. $2 \mathrm{~A}$ and B). The percentage of vessel area per field was significantly higher in the VEGF+apelin group than in the LacZ group $(\mathrm{p}<0.05$; Fig. 2C). Moreover, the number of large ( $>10 \mu \mathrm{m})$ vessels in the VEGF+apelin group was significantly higher than the number found in the LacZ, VEGF, and apelin groups ( $p<0.05$, respectively; Fig. 2D).

Protein levels of VEGF and apelin in the attached muscle and cortex 1 week after EMS were evaluated in all 4 groups. VEGF protein levels in the attached muscle tended to be higher in the VEGF and VEGF+apelin groups than in the other groups, but the differences were not statistically significant ( $p=0.095$, single ANOVA). VEGF protein was not detected in the cortex for any of the groups (Fig. 3A). Apelin protein was detected in the attached muscle only in the apelin group and the VEGF+apelin group. Apelin protein was not detected in the cortex for any of the groups (Fig. 3B).

Immunohistochemical staining of brain tissue harvested 7 days after CCAO with monoclonal anti-apelin antibody and polyclonal anti-APJ antibody revealed that ECs stained by RECA-1 or von Willenbrand factor antibody in the cortex express apelin or APJ (Fig. 4).

\section{Discussion}

\section{Indirect Bypass Surgery for Moyamoya Disease}

Direct and/or indirect bypass surgery is often performed as a treatment for patients with MMD. Although direct bypass surgeries such as superficial temporal artery-middle cerebral artery anastomosis are frequently performed in adult and pediatric patients, they are sometimes difficult, especially in young children. Given that indirect bypass surgery is easy and simple to perform, it is a commonly used method to increase CBF in patients with MMD. Although indirect bypass surgeries are effective for pediatric and young adult patients with MMD, direct bypass surgery remains the main treatment option for most adults with MMD. ${ }^{27}$ This is because indirect bypass surgery often results in insufficient collateral circulation for most adult and some pediatric patients..$^{20,25,27,32,39} \mathrm{An}$ endogenous angiogenic factor may be involved in the induction of blood vessel growth associated with collateral circulation. Park et al. demonstrated that the genotype of the VEGF allele was related to better collateral vessel formation after bypass surgery in patients with MMD. ${ }^{33}$ These data indicate that the addition of an exogenous an- 


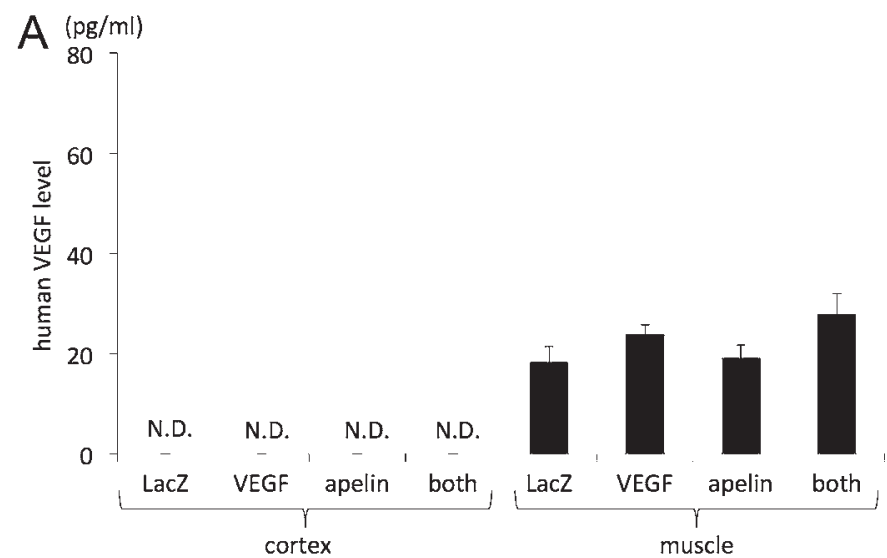

ANOVA: $p=0.095$

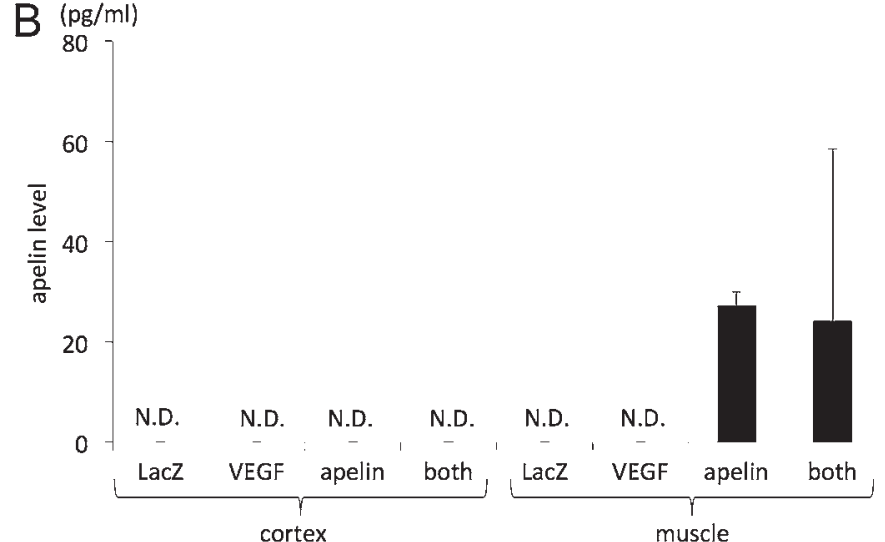

ANOVA: $p=0.28$

FIG. 3. ELISA results for human VEGF and apelin. A: Human VEGF levels in the cortex and attached muscle 1 week after indirect bypass surgery and injection of plasmids. B: Apelin levels in the cortex and attached muscle 1 week after indirect bypass surgery and injection of plasmids. N.D. = not detected.

giogenic factor to indirect bypass surgery could enhance the number and growth of collateral vessels.

\section{Gene Therapy in a Chronic Cerebral Hypoperfusion Model}

In this report, we confirmed that the total number of blood vessels in the cortex was significantly larger in the VEGF group and the VEGF+apelin group than in the LacZ group. Moreover, larger vessels were induced in the VEGF+apelin group than in the other groups. In the hypoperfusion model, indirect bypass surgery without angiogenic factors could increase the number of vessels, and the presence of additional angiogenic factors could

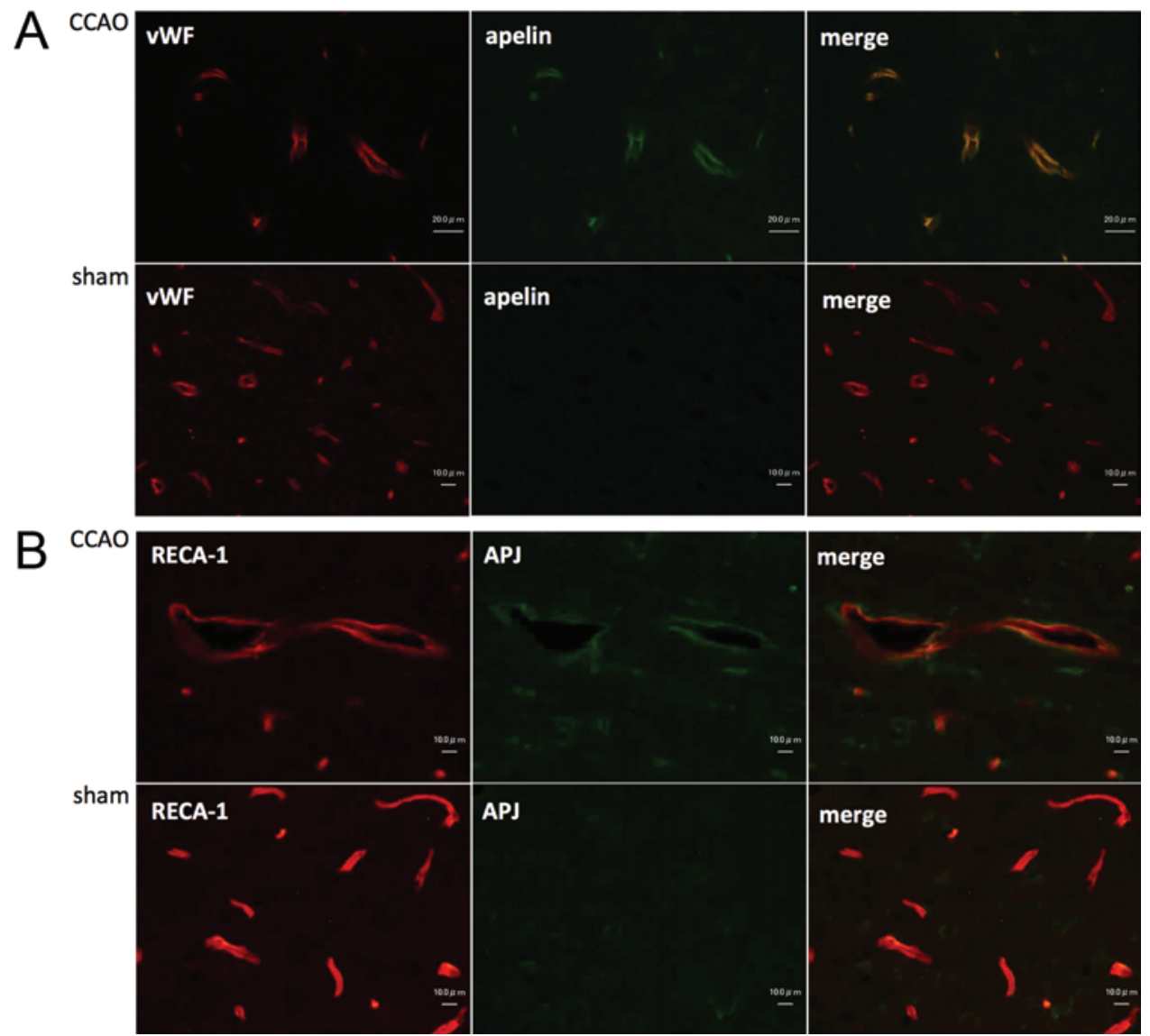

FIG. 4. Immunohistochemical analysis of cortex tissue from CCAO and sham-operated (sham) rats. A: Immunohistochemical staining using anti-apelin antibody. B: Immunohistochemical staining using anti-APJ antibody. vWF = anti-von Willebrand factor antibody. 
lead to a further increase. $1,2,12,15,19,22,31$ Hecht et al. showed that combining EMS with VEGF-expressing myoblasts in mice that had undergone unilateral internal carotid artery ligation improved not only the vessel density of the cortex but also the cerebrovascular reserve capacity. ${ }^{12}$ However, similar reports investigating vessel caliber size are limited. Ohmori et al. reported that combining encephalogaleosynangiosis (EGS) with the intramuscular injection of recombinant granulocyte-colony stimulating factor (G-CSF) in hypoperfusion rats increased the number of smaller vessels. ${ }^{31}$ We thought that the increase in the number of larger vessels was an important factor in mature angiogenic development. Kidoya et al. reported that the apelin/APJ system spatially and temporally modulates enlargement of vessel diameter during embryogenesis. ${ }^{18}$ Several reports suggest that apelin expression is induced in ECs during hypoxia., ${ }^{7,14,17,18}$ The mechanism by which enlarged blood vessels are produced by the apelin/APJ system has been described by Takakura and Kidoya. ${ }^{36}$ VEGF and angiopoietin-1 (Ang-1) induce expression of APJ and apelin on ECs. Under the expression of VEGF, apelin promotes the aggregation and proliferation of ECs, which lead to mature angiogenesis, such as caliber size enlargement. When caliber size becomes sufficient to supply oxygen to the tissue, angiogenesis factors will be downregulated and the regulation of caliber size is completed.

Newly formed vessels induced by overexpression of VEGF alone can be immature and are at risk for tissue edema $^{11}$ or hemangioma. ${ }^{35}$ Therefore, several groups have reported on the use of combined gene therapy, namely VEGF plus Ang-1 for ischemic hindlimb models, myocardial infarction models, or acute cerebral ischemia models and VEGF plus apelin for ischemic hindlimb models. . $, 5,17,34,40,41$ The merits of VEGF plus apelin combined gene therapy were reported to include increases in vessel density and the maturation of newly developed vessels, such as larger vessel formation and low permeability. ${ }^{36}$ Some studies have reported that VEGF-mediated permeability occurs through the disorganization of endothelial junction proteins, such as vascular endothelial (VE)-cadherin. ${ }^{10,16}$ It was also reported that apelin inhibited the down-modulation of VE-cadherin by VEGF, resulting in the suppression of hyperpermeability. ${ }^{17}$ Although we observed the formation of larger vessels, we cannot confirm the suppression of hyperpermeability in the VEGF+apelin group. We think that when we add gene therapy to the indirect bypass method, the development of mature, newly formed vessels due to the bypass surgery itself may lead to a reduction in adverse effects and an increase in CBF.

In the current study, secreted proteins were detected only in muscles, and this has also been described in a previous report by our group.22 We thought that the injection of VEGF plus apelin plasmids into the muscle would increase angiogenesis not only in the muscle but also in the muscle-cortex interface. Then, the transpial vessel sprouted into the cortex. Similar models have demonstrated this mechanism. ${ }^{12,28}$ In particular, Nakamura et al. showed the mechanism of revascularization in an experimental model using EMS in pigs. ${ }^{28}$ During cerebral ischemia, the infiltration of inflammatory cells between the temporal muscle and the arachnoid membrane induced angiogenesis and led to revascularization between the external cerebral artery and the cerebral cortex artery.

\section{Study Limitations}

This study has several limitations. First, the observational periods of the immunohistochemical analyses and protein assay were short. Our previous studies and similar studies from other institutions have reported results from evaluations between 2 and 4 weeks.,12,15,19,22,31 In general, angiogenesis occurs over several months after indirect bypass surgery in patients with MMD. Future studies need to analyze collateral formation and protein levels in this model over a longer period.

Second, we could not conduct behavioral assessments in our model. Cognitive impairment can occur in patients with MMD., ${ }^{9} 13$ Assessing the correlation between development of collateral formation and change in cognitive function in this model is desirable.

Third, we did not measure vessel dysfunction, such as breakdown of the blood-brain barrier, vessel leakiness, tight junction protein assessment, or brain edema.

Fourth, we could not conduct blood flow measurements. In previous reports by other groups, CCAO in rats reduced CBF to $35 \%-50 \%$ of control levels. ${ }^{8,37}$ In the future, we need to measure blood flow to assess the effect of enhanced angiogenesis.

Therefore, further analyses such as those described above are needed before applying the described technique in the clinical setting.

\section{Conclusions}

Combining EMS with gene therapy (VEGF plus apelin) in a chronic cerebral hypoperfusion model in rats can enhance mature angiogenesis. It could be a feasible treatment option in the clinical setting for patients with MMD.

\section{Acknowledgments}

We thank Dr. H. Kidoya for supplying VEGF, apelin, and LacZ plasmids and Dr. H. Michiue, Ms. A. Ueda, Dr. T. Oohashi, Ms. A. Maehara, Ms. M. Arao, and Ms. N. Uemori for technical assistance.

\section{References}

1. Anan M, Abe T, Matsuda T, Ishii K, Kamida T, Fujiki M, et al: Induced angiogenesis under cerebral ischemia by cyclooxygenase 2 and hypoxia-inducible factor naked DNA in a rat indirect-bypass model. Neurosci Lett 409:118-123, 2006

2. Anan M, Abe T, Shimotaka K, Kamida T, Kubo T, Fujiki M, et al: Induction of collateral circulation by hypoxia-inducible factor 1alpha decreased cerebral infarction in the rat. Neurol Res 31:917-922, 2009

3. Arsic N, Zentilin L, Zacchigna S, Santoro D, Stanta G, Salvi A, et al: Induction of functional neovascularization by combined VEGF and angiopoietin-1 gene transfer using AAV vectors. Mol Ther 7:450-459, 2003

4. Baumgartner I, Rauh G, Pieczek A, Wuensch D, Magner M, Kearney M, et al: Lower-extremity edema associated with gene transfer of naked DNA encoding vascular endothelial growth factor. Ann Intern Med 132:880-884, 2000 
5. Chae JK, Kim I, Lim ST, Chung MJ, Kim WH, Kim HG, et al: Coadministration of angiopoietin-1 and vascular endothelial growth factor enhances collateral vascularization. Arterioscler Thromb Vasc Biol 20:2573-2578, 2000

6. Dai T, Ramirez-Correa G, Gao WD: Apelin increases contractility in failing cardiac muscle. Eur J Pharmacol 553:222-228, 2006

7. Eyries M, Siegfried G, Ciumas M, Montagne K, Agrapart M, Lebrin F, et al: Hypoxia-induced apelin expression regulates endothelial cell proliferation and regenerative angiogenesis. Circ Res 103:432-440, 2008

8. Farkas E, Luiten PG, Bari F: Permanent, bilateral common carotid artery occlusion in the rat: a model for chronic cerebral hypoperfusion-related neurodegenerative diseases. Brain Res Brain Res Rev 54:162-180, 2007

9. Festa JR, Schwarz LR, Pliskin N, Cullum CM, Lacritz L, Charbel FT, et al: Neurocognitive dysfunction in adult moyamoya disease. J Neurol 257:806-815, 2010

10. Gavard J, Gutkind JS: VEGF controls endothelial-cell permeability by promoting the beta-arrestin-dependent endocytosis of VE-cadherin. Nat Cell Biol 8:1223-1234, 2006

11. Harrigan MR, Ennis SR, Masada T, Keep RF: Intraventricular infusion of vascular endothelial growth factor promotes cerebral angiogenesis with minimal brain edema. Neurosurgery 50:589-598, 2002

12. Hecht N, Marushima A, Nieminen M, Kremenetskaia I, von Degenfeld G, Woitzik J, et al: Myoblast-mediated gene therapy improves functional collateralization in chronic cerebral hypoperfusion. Stroke 46:203-211, 2015

13. Karzmark P, Zeifert PD, Tan S, Dorfman LJ, Bell-Stephens TE, Steinberg GK: Effect of moyamoya disease on neuropsychological functioning in adults. Neurosurgery 62:1048-1052, 2008

14. Kasai A, Shintani N, Oda M, Kakuda M, Hashimoto H, Matsuda T, et al: Apelin is a novel angiogenic factor in retinal endothelial cells. Biochem Biophys Res Commun 325:395-400, 2004

15. Katsumata A, Sugiu K, Tokunaga K, Kusaka N, Watanabe K, Nishida A, et al: Optimal dose of plasmid vascular endothelial growth factor for enhancement of angiogenesis in the rat brain ischemia model. Neurol Med Chir (Tokyo) 50:449-455, 2010

16. Kevil CG, Payne DK, Mire E, Alexander JS: Vascular permeability factor/vascular endothelial cell growth factormediated permeability occurs through disorganization of endothelial junctional proteins. J Biol Chem 273:1509915103, 1998

17. Kidoya H, Naito H, Takakura N: Apelin induces enlarged and nonleaky blood vessels for functional recovery from ischemia. Blood 115:3166-3174, 2010

18. Kidoya H, Ueno M, Yamada Y, Mochizuki N, Nakata M, Yano T, et al: Spatial and temporal role of the apelin/APJ system in the caliber size regulation of blood vessels during angiogenesis. EMBO J 27:522-534, 2008

19. Kim HS, Lee HJ, Yeu IS, Yi JS, Yang JH, Lee IW: The neovascularization effect of bone marrow stromal cells in temporal muscle after encephalomyosynangiosis in chronic cerebral ischemic rats. J Korean Neurosurg Soc 44:249255, 2008

20. Kim SK, Cho BK, Phi JH, Lee JY, Chae JH, Kim KJ, et al: Pediatric moyamoya disease: An analysis of 410 consecutive cases. Ann Neurol 68:92-101, 2010

21. Kuroda S, Houkin K: Moyamoya disease: current concepts and future perspectives. Lancet Neurol 7:1056-1066, 2008

22. Kusaka N, Sugiu K, Tokunaga K, Katsumata A, Nishida A, Namba K, et al: Enhanced brain angiogenesis in chronic cerebral hypoperfusion after administration of plasmid human vascular endothelial growth factor in combination with indirect vasoreconstructive surgery. J Neurosurg 103:882-890, 2005

23. Lee DK, Cheng R, Nguyen T, Fan T, Kariyawasam AP, Liu Y, et al: Characterization of apelin, the ligand for the APJ receptor. J Neurochem 74:34-41, 2000

24. Lee RJ, Springer ML, Blanco-Bose WE, Shaw R, Ursell PC, Blau HM: VEGF gene delivery to myocardium: deleterious effects of unregulated expression. Circulation 102:898-901, 2000

25. Lee SB, Kim DS, Huh PW, Yoo DS, Lee TG, Cho KS: Longterm follow-up results in 142 adult patients with moyamoya disease according to management modality. Acta Neurochir (Wien) 154:1179-1187, 2012

26. Masri B, Morin N, Cornu M, Knibiehler B, Audigier Y: Apelin (65-77) activates p70 S6 kinase and is mitogenic for umbilical endothelial cells. FASEB J 18:1909-1911, 2004

27. Mizoi K, Kayama T, Yoshimoto T, Nagamine Y: Indirect revascularization for moyamoya disease: is there a beneficial effect for adult patients? Surg Neurol 45:541-549, 1996

28. Nakamura M, Imai H, Konno K, Kubota C, Seki $\mathrm{K}$, Puentes S, et al: Experimental investigation of encephalomyosynangiosis using gyrencephalic brain of the miniature pig: histopathological evaluation of dynamic reconstruction of vessels for functional anastomosis. Laboratory investigation. J Neurosurg Pediatr 3:488-495, 2009

29. O'Carroll AM, Selby TL, Palkovits M, Lolait SJ: Distribution of mRNA encoding B78/apj, the rat homologue of the human APJ receptor, and its endogenous ligand apelin in brain and peripheral tissues. Biochim Biophys Acta 1492:72-80, 2000

30. O'Donnell LA, Agrawal A, Sabnekar P, Dichter MA, Lynch DR, Kolson DL: Apelin, an endogenous neuronal peptide, protects hippocampal neurons against excitotoxic injury. J Neurochem 102:1905-1917, 2007

31. Ohmori Y, Morioka M, Kaku Y, Kawano T, Kuratsu $\mathrm{J}$ : Granulocyte colony-stimulating factor enhances the angiogenetic effect of indirect bypass surgery for chronic cerebral hypoperfusion in a rat model. Neurosurgery 68:1372-1379, 2011

32. Pandey P, Steinberg GK: Outcome of repeat revascularization surgery for moyamoya disease after an unsuccessful indirect revascularization. Clinical article. J Neurosurg 115:328336, 2011

33. Park YS, Jeon YJ, Kim HS, Chae KY, Oh SH, Han IB, et al: The role of VEGF and KDR polymorphisms in moyamoya disease and collateral revascularization. PLoS One 7:e47158, 2012

34. Samuel SM, Akita Y, Paul D, Thirunavukkarasu M, Zhan L, Sudhakaran PR, et al: Coadministration of adenoviral vascular endothelial growth factor and angiopoietin-1 enhances vascularization and reduces ventricular remodeling in the infarcted myocardium of type 1 diabetic rats. Diabetes 59:51-60, 2010

35. Schwarz ER, Speakman MT, Patterson M, Hale SS, Isner JM, Kedes LH, et al: Evaluation of the effects of intramyocardial injection of DNA expressing vascular endothelial growth factor (VEGF) in a myocardial infarction model in the ratangiogenesis and angioma formation. J Am Coll Cardiol 35:1323-1330, 2000

36. Takakura N, Kidoya H: Maturation of blood vessels by haematopoietic stem cells and progenitor cells: involvement of apelin/APJ and angiopoietin/Tie2 interactions in vessel caliber size regulation. Thromb Haemost 101:999-1005, 2009

37. Tanaka K, Ogawa N, Asanuma M, Kondo Y, Nomura M: Relationship between cholinergic dysfunction and discrimination learning disabilities in Wistar rats following chronic cerebral hypoperfusion. Brain Res 729:55-65, 1996

38. Tashiro H, Aoki M, Isobe M, Hashiya N, Makino H, Kaneda 
Y, et al: Development of novel method of non-viral efficient gene transfer into neonatal cardiac myocytes. J Mol Cell Cardiol 39:503-509, 2005

39. Touho H, Karasawa J, Ohnishi H, Yamada K, Shibamoto $\mathrm{K}$ : Surgical reconstruction of failed indirect anastomosis in childhood Moyamoya disease. Neurosurgery 32:935-940, 1993

40. Toyama K, Honmou O, Harada K, Suzuki J, Houkin K, Hamada $\mathrm{H}$, et al: Therapeutic benefits of angiogenetic genemodified human mesenchymal stem cells after cerebral ischemia. Exp Neurol 216:47-55, 2009

41. Yamauchi A, Ito Y, Morikawa M, Kobune M, Huang J, Sasaki K, et al: Pre-administration of angiopoietin-1 followed by VEGF induces functional and mature vascular formation in a rabbit ischemic model. J Gene Med 5:994-1004, 2003

\section{Disclosures}

The authors report no conflict of interest concerning the materi- als or methods used in this study or the findings specified in this paper.

\section{Author Contributions}

Conception and design: Hiramatsu, Tokunaga, Sugiu, Date. Acquisition of data: Hiramatsu, Nishihiro. Analysis and interpretation of data: Hiramatsu, Nishihiro, Haruma, Shimizu, Takasugi, Shinji. Drafting the article: Hiramatsu, Hishikawa, Date. Critically revising the article: Hiramatsu, Hishikawa. Reviewed submitted version of manuscript: all authors. Approved the final version of the manuscript on behalf of all authors: Hiramatsu. Statistical analysis: Hiramatsu. Administrative/technical/material support: Kidoya, Takakura. Study supervision: Hishikawa, Tokunaga, Kidoya, Sugiu, Takakura.

\section{Correspondence}

Masafumi Hiramatsu, Department of Neurological Surgery, Okayama University Graduate School of Medicine, Dentistry and Pharmaceutical Sciences, 2-5-1 Shikata-cho, Kita-ku, Okayama 700-8558, Japan. email: mhiramatsu@okayama-u.ac.jp. 